\title{
Industrial Burner Modeling: Final Report for the CIEE
}

\author{
L.D. Cloutman
}

December 1994

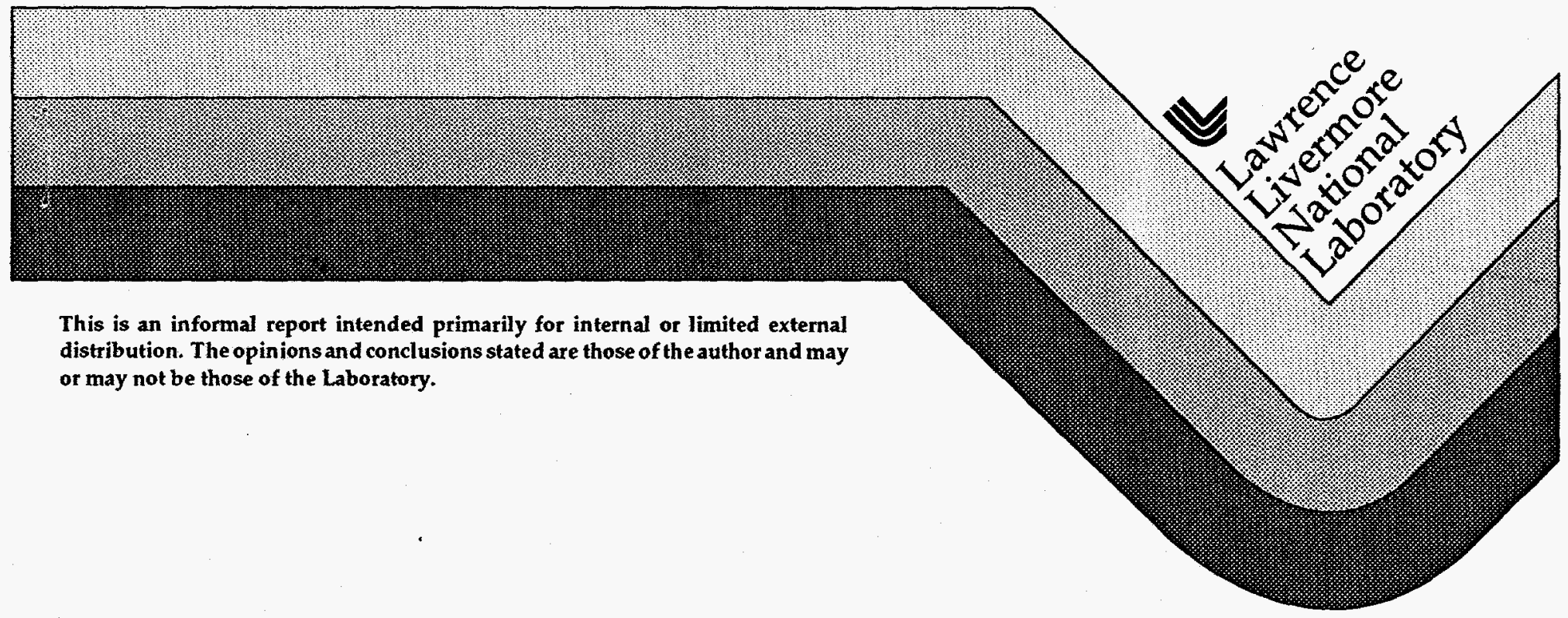

DISTPIBUTION OF THIS DOCUMENT IS UNLIMITED 35 


\section{DISCLAIMER}

Portions of this document may be illegible in electronic image products. Images are produced from the best available original document. 


\title{
INDUSTRIAL BURNER MODELING: FINAL REPORT FOR THE CIEE
}

\author{
L. D. Cloutman \\ H Division, L-298 \\ Lawrence Livermore National Laboratory \\ Livermore, California
}

\begin{abstract}
The COYOTE computer program was used as the basis for a comprehensive numerical model of industrial burners. This program is based on the full multicomponent Navier-Stokes equations and includes a subgrid-scale turbulence model. The model was used to simulate the flows in a laboratory-scale burner that is being studied experimentally at UC-Irvine. We summarize what has been learned in the last three years from simulations of this burner. We demonstrate that this model provides detailed information about the flow field in the furnace, making it a useful tool for studying the physics of burners.
\end{abstract}




\section{INTRODUCTION}

Numerical simulation of industrial burners is a challenging computational problem. As part of a systematic study of this problem, we engaged in a screening study of a simplified burner that was proposed for experimental investigation at the University of California at Irvine for the California Institute for Energy Efficiency (CIEE). The long-range goals of this study are to identify parameters that affect the production of $\mathrm{NO}_{\mathrm{x}}$ and to discover methods of reducing emissions while maintaining or improving efficiency. This report describes the progress we have made toward these goals and documents what we have learned about the physics of burners.

We used the COYOTE numerical fluid dynamics program [1], which was designed to simulate reacting flows in two-dimensional Cartesian and axisymmetric geometries. The current version of the program has a variety of turbulence models, including the LUVD11 subgrid-scale (SGS) turbulence model [2] and the $k-\epsilon$ model [3-5].

COYOTE is based on the full transient Navier-Stokes equations for a reactive multicomponent gas mixture. Steady-state solutions, when they exist, are found by assuming an arbitrary initial flow and allowing the transient to decay. While this approach is less efficient computationally than a direct steady state approach, it has several advantages. First, it does not make any ad hoc assumption that the final flow field is truly steady. That is, it allows the final solution to contain quasi-periodic features such as vortex shedding or flame flickering. If the final flow is truly steady, then that type of solution will evolve naturally. Second, it automatically performs a flame stability study. If, for example, the flame cannot be sustained, it should be extinguished in the calculation (at least to the extent that the gas physics included in the calculation is an accurate representation of the physical system). Third, it allows easy incorporation of complex gas physics, such as detailed chemical kinetics or radiative heat losses. The main disadvantage is that the computational expense is higher than for a direct steady-state solver. However, in today's world of relatively abundant and inexpensive computational power and high personnel costs, we can afford to trade a certain amount of computational effort (in both algorithmic efficiency and the inclusion 
of physical detail) for a reduction in the human effort required to obtain solutions.

The burner is axisymmetric and includes a large enclosure to confine the flame. This is the same experimental arrangement as used for a set of cold flow simulations [6]. The experimental nozzle and flame zone are confined inside a combustion chamber, which is a octagonal prism approximated by a cylinder $30.48 \mathrm{~cm}$ in radius and $93.98 \mathrm{~cm}$ tall above the entrance plane of the fuel nozzle. The chamber is $91.44 \mathrm{~cm}$ tall above the top of the quarl, and it is topped by a roof with a slope of $45^{\circ}$ that necks down to fit a $15.24 \mathrm{~cm}$ diameter chimney. Preliminary calculations covering only the corner of the furnace near the nozzles showed that it was necessary to include the whole furnace in the computational domain to avoid numerical difficulties with the outflow boundaries [7]. Therefore the present computational mesh fills this enclosure, so the walls are approximated by a fixed-temperature boundary with heat transfer and drag computed from a lawof-the-wall model. A fixed pressure subsonic outflow boundary is used at the opening on the top of the grid. All calculations place the bottom of the computational grid at the exit plane of the fuel nozzle. The flow conditions are for a heating rate of $29.3 \mathrm{~kW}\left(1.0 \times 10^{5} \mathrm{Btu} / \mathrm{hr}\right)$. A more detailed description of the geometry and boundary conditions may be found in earlier reports $[6,7]$.

Section II describes the geometry of the experimental apparatus and the problem setup. Section III describes three reactive flow solutions for this burner. Conclusions are presented in Section IV.

\section{PROBLEM DESCRIPTION}

COYOTE is based on the full Navier-Stokes equations for a mixture of compressible gases. The equations, turbulence model, and numerical algorithms have been described in detail elsewhere $[1,2]$ and will not be repeated here. Species diffusion is modeled by Fick's law and the heat flux is modeled by Fourier's law plus enthalphy diffusion. The thermal equation of state is the sum of partial pressures for each species treated as an ideal gas. The species internal energies are functions of temperature taken from the JANAF tables [8-10]. The molecular viscosity of the mixture is 
assumed to be given by

$$
\mu=1.457 \times 10^{-5} T^{3 / 2} /(110+T),
$$

where $T$ is the absolute temperature. The thermal conductivity is calculated from the viscosity using a constant Prandtl number with a value of 0.7 . The species diffusivities are assumed to be equal and are calculated from the viscosity and a constant Schmidt number, also 0.7.

The chemical reaction network consists of a single global reaction for methane oxidation to $\mathrm{H}_{2} \mathrm{O}$ and $\mathrm{CO}_{2}$, the extended Zel'dovich mechanism for thermal $\mathrm{NO}$ production, and six molecular equilibrium reactions for the dissociation of $\mathrm{H}_{2} \mathrm{O}, \mathrm{CO}_{2}, \mathrm{H}_{2}, \mathrm{O}_{2}, \mathrm{~N}_{2}$, and $\mathrm{OH}$. The reactions are listed in Table 1. The fuel oxidation reaction rate was inspired by the work of Westbrook and Dryer [11], and we used

$$
k_{\mathrm{ov}}=9.5 \times 10^{14}\left[\mathrm{CH}_{4}\right]^{0.25}\left[\mathrm{O}_{2}\right]^{1.5} \exp (-24358 / T)
$$

The computational mesh extends from the axis of symmetry on the left to the walls of the furnace on the right, from the exit plane of the nozzle at the bottom to the base of the chimney at the top. Obstacles and the sloping roof are formed by blocking out appropriate computational zones. We use a 125 by 287 grid. The zones near the nozzle are $0.079 \mathrm{~cm}$ square at the lower left corner, growing slightly to $\delta x=0.653 \mathrm{~cm}$ and $\delta y=0.609 \mathrm{~cm}$ at the upper right corner.

\section{NUMERICAL REACTIVE FLOW SOLUTIONS}

Figure 1 shows some typical results of cold flow calculations. The center panel is the streamline plot for the calculation made using the entire furnace. The left panel is the same problem, but with the grid truncated at the base of the sloping roof. The right panel is the velocity vector plot corresponding to the streamlines in the center panel. There are two important features to note. First, the presence of the sloping roof makes a huge difference in the stream function, affecting the spatial distribution and the amplitude. Second, the dominant flow feature in the velocity field is the axial jet extending from the bottom of the quarl to the chimney. The velocity vectors for the 
left panel are essentially identical to those shown on the right. This means that the part of the flow away from the axis is very slow but producing a significant mass flow rate and sensitive to the presence of the roof. This figure shows that quantities such as the residence time in the outer part of the furnace and the precise composition of material recirculated back into the flame zone (in the reactive case) may be very sensitive to the precise domain of integration and to the boundary conditions on that domain. Runs made with and without the wall function boundary layer model show a similar degree of sensitivity.

Another cold flow problem that we are in the process of solving is in the swirl air supply chamber below the quarl. This calculation is shown in Fig. 2. The left panel extends from the bottom of the chamber to the top opening of the quarl. The left boundary is the axis of symmetry. Part of the air flows into the chamber through an annulus next to the fuel pipe, which is the obstacle along the axis. The rest of the air flows in through tangential jets approximately half way up the chamber walls. In the calculation, which is axisymmetric, the inflow from the tangential jets is modeled with volumetric source terms for mass, heat, and swirl velocity. The degree of swirl is determined by the ratio of these two flow rates. What is needed for the burner simulations is the swirl velocity distribution at the bottom of the quarl, and that is the reason for this type of simulation. We began this calculation so recently that this first case is not yet finished, but we show it to demonstrate our capabilities.

The base case for the reactive flow calculations uses a cone annular fuel nozzle. The isotherms are shown in Fig. 3. The flame is confined to the rounded region in the lower left-hand corner and is stabilized by air swirl. Figure 4 shows the $12 \mathrm{~cm}$ square region around the flame. Burning fuel and air flow up and to the right along the face of the quarl. Some of the hot products continue upward and out the chimney, and the rest are recirculated downward along the axis, stabilizing the flame. Figure 5 shows the rate of production on NO, which correlates well with high temperatures as expected. Figure 6 shows contours of NO mass fraction. The peak NO mass fraction is in the center of the vortex that defines the recirculation zone. The maximum value at the particular time of this snapshot is on the axis at the top opening of the quarl. The reactive flows all show a large 
degree of time dependence, and this atypical peak later disappears. However, the relatively low values of NO higher up along the axis are a permanent feature and are in qualitative agreement with the LIF measurements, which show the peak NO abundance is off-axis. A convincing explanation of the NO spatial distribution is not available yet.

Figure 7 shows the time evolution of the isotherms for the same burner except there is no swirl. Experimentally, this situation is known to be unstable and cannot sustain the flame. In the calculation, there was difficulty in establishing the flame, which stands off the burner as shown in the left panel. The flame is the short horizontal contour intersecting the axis several centimeters above the nozzle. There is no recirculation zone except for an insignificant vortex ring in the wake of the cone of the fuel nozzle. This configuration appeared to be nearly stable, but as shown in the next two panels, the flame slowly began lifting, and the rate liftoff kept increasing. Eventually the flame went out and the hot gases were expelled from the grid.

Another variation of the base case burner with swirl is shown in Fig. 8. The axial velocity of the swirl air was modulated at $250 \mathrm{~Hz}$ with a 10 percent amplitude. The effect on the flame is profound. First, the flame sheet separates from the face of the quarl so the included angle of the flame is closer to 40 degrees than 90 . Second, the smooth flow along the flame sheet breaks up into a vortex street. Four of these small vortices are seen just above the nozzle. The large single vortex in the upper half of the plot is due to a $10 \mathrm{~Hz}$ Kelvin-Helmholtz instability that is generated along the outer edge of the air stream in all of the reactive flow cases. This solution illustrates the potential of acoustic modes to have pronounced effects on the flame.

Figure 9 shows the isotherms for two solutions with an axial fuel injection nozzle. The left panel is for the laboratory-scale furnace used in all of the previous solutions. The right panel shows the solution for a furnace with all lengths multiplied by a factor of five. Fluid velocities remain the same, so this case has 25 times the power of the smaller furnace. As with the cone-annular nozzle, the solution shows a strong recirculation zone inside the flame sheet, and a series of vortices are shed along the edge of the air stream at about $10 \mathrm{~Hz}$. In the cone-annular case, the recirculation zone produces downflow along the axis all the way down to the fuel nozzle. In this case, the axial 
fuel jet produces upflow along the axis for several centimeters. In the cone-annular case, the fuel and air are nearly completely mixed inside the quarl. With the axial injector, the fuel jet extends several centimeters above the quarl. The poor mixing is expected to produce poor performance in both efficiency and emissions.

The large-scale solution is qualitatively the same in spite of a factor of five increase in the Reynolds number. There are two differences. First, the large-scale solution shows a significantly cooler flame, which is helpful in reducing thermal NO. Second, the flame zone is smaller relative to the size of the furnace in the large-scale solution. This can be seen by comparing the location of the right edge of the flame sheet to the location of the secondary air channel.

Figure 10 shows isotherms for the laboratory-scale burner with and without the turbulence model. The fuel jet essentially a round turbulent jet and should have a structure similar to the selfsimilar solution for such a jet. The solutions in Fig. 9 and in the right-hand panel of Fig. 10 fulfill this expectation. The jet spreads at the correct rate, and the axial velocity drops approximately inversely with the distance from the injector. Without the turbulence model, the fuel jet does not spread at all, and the velocity is independent of axial position. The inflowing fuel heads straight for the chimney, mixing and burning very little with the air. It is doubtful that this flame is stable. The problem is that the zoning is much coarser than the Kolmogorov microscale, and the unresolved scales are important to the dynamics of the jet. This result casts serious doubt on the usefulness of the practice of simply ignoring the subgrid scales, a practice occasionally encountered in the literature. The danger is that one can create plausible, but very wrong, solutions unless all of the relevant physics is included.

Figure 11 shows the axial temperature distribution and axial NO mass flow rate in a small combustor $[12,13]$. The curves labelled with an $\mathrm{A}$ ignore radiative heat losses from the gas, and those labelled with a $\mathrm{B}$ made use of a simple radiative heat loss model [14]. The solid circles in the temperature plot are experimental data points with an uncertainty of approximately 100 K. The radiative losses improve the agreement with experiment. The heat losses make a 50 to $100 \mathrm{~K}$ difference at most locations in the burner, and this makes a factor of two difference in the 
amount of thermal NO leaving the combustor. We conclude that accurate predictions of emissions requires knowledge of the temperature field to better than $50 \mathrm{~K}$, and that radiative transfer must be included in a realistic manner.

\section{CONCLUSIONS AND FUTURE TASKS}

The COYOTE hydrodynamics program has been used as the basis of a comprehensive numerical model designed to simulate flows in industrial burners. The primary objectives of this study are 1) validation of the program and the turbulence models by applying them to a simplified experimental burner and 2) to study the physics of burner operation. Numerical solutions have led to several conclusions:

1) Detailed simulations are difficult and costly, but they have a big payoff in terms of the large amount of physical information provided. We have demonstrated the feasibility of using scientific workstations to produce multidimensional simulations with 13 species, 10 chemical reactions, a subgrid-scale turbulence model, and a simple radiative heat loss model as a research tool to provide a few well-resolved solutions to study burner physics.

2) The solutions are very sensitive to boundary conditions, a consequence of the elliptic nature of the partial differential equations. This means that good numerical algorithms for the inflow and outflow boundaries are needed, and boundary layer drag and heat transfer must be included on all solid surfaces. Furthermore, the entire furnace must be included in the calculation because boundary effects propagate all the way across the mesh.

3) We have identified important physical processes that must be included in the model. These include boundary layer effects, a turbulence model to account for unresolved flow features, a sufficiently detailed chemical kinetics mechanism, real gas physics (caloric equation of state and transport coefficients), and radiative heat transfer.

4) Cold and reactive flows are fundamentally different. Not only are the flow profiles different, the cold flows tend to be steady whereas the reactive flows are all oscillatory in time. 
5) The temperature field must be computed quite accurately if accurate predictions of NO emissions are to be made. The high temperature dependence of the thermal NO mechanism means that an error of $50 \mathrm{~K}$ in the combustion products is significant.

6) Mixing occurs very close to the injector. Turbulence levels are high only near the injector, suggesting that we need to include the molecular transport coefficients in a large part of the enclosure, a task that has been done [15].

In addition, we have identified a number of tasks remaining to be accomplished in the future:

1) More detailed comparisons with experiments are needed both to validate the model and to interpret the results of the experiments.

2) We need to complete a project to improve the fuel oxidation mechanisms.

3) The nitrogen oxide mechanisms need more work. Thermal NO is in good shape, but prompt and nitrous-route NO must be included.

4) We need to improve the turbulent mixing model. The flux-gradient approximation is questionable.

5) The turbulence-chemistry closure problem must be addressed.

6) The radiative transfer model must be improved.

\section{ACKNOWLEDGMENTS}

I thank Charles Westbrook, Scott Samuelsen, Bill Sowa, and Matt Miyasato for helpful conversations in defining the problem. I also thank Lila Chase, Bill Pitz, and Jay McGowan for their aid in making COYOTE a functional tool on a variety of computing platforms. Thanks also to Leigh Brookshaw for his help in speeding up the code and in producing improved graphical output. Special thanks to Matt Miyasato for an enlightening demonstration of the qualitative flow features of the flame in this burner. Finally, I thank Tom McAbee for communicating his experience with the turbulence model. This work was supported by the California Institute for Energy Efficiency and Southern California Gas Company. This work was performed under the auspices of the U.S. 
Department of Energy and the Lawrence Livermore National Laboratory under contract number W-7405-ENG-48.

\section{REFERENCES}

1. Cloutman, L. D., "COYOTE: A Computer Program for 2-D Reactive Flow Simulations," Lawrence Livermore National Laboratory report UCRL-ID-103611, 1990.

2. Cloutman, L. D., "The LUVD11 Large Eddy Simulation Model," Lawrence Livermore National Laboratory report UCRL-ID-107128, 1991.

3. Launder, B. E. and Spalding, D. B., Mathematical Models of Turbulence (London: Academic Press), 1972.

4. Launder, B. E. and Spalding, D. B. 1974, Comp. Meth. App. Mech. Eng., 3, 269.

5. Jones, W. P., in Prediction Methods for Turbulent Flows, ed. W. Kollmann (Washington: Hemisphere), 1980, p. 379.

6. L. D. Cloutman, "Numerical Simulation of Cold Flow Patterns and Turbulent Mixing in a Simplied Burner", Lawrence Livermore National Laboratory report UCRL-ID-118861, 1994.

7. Cloutman, L. D., "Numerical Simulation of Turbulent Mixing and Combustion Near the Inlet of a Burner," Lawrence Livermore National Laboratory report UCRL-JC-112943, 1993.

8. Stull, D. R. and Prophet, H., JANAF Thermochemical Tables, 2nd ed. (U. S. Department of Commerce/National Bureau of Standards, NSRDS-NBS 37, June 1971).

9. Chase, M. W., Curnutt, J. L., Hu, A. T., Prophet, H., Syverud, A. N., and Walker, L. C. 1974, JANAF Thermochemical Table, 1974 Supplement, J. Phys. Chem. Ref. Data, $3,311$.

10. Chase, M. W. Jr., Davies, C. A., Downey, J. R. Jr., Frurip, D. J., McDonald, M. A., and Syverud, A. N. 1985, JANAF Thermochemical Tables, Third Edition, Parts I and II. Supplement No. 1. J. Phys. Chem. Ref. Data, 14.

11. Westbrook, C. K. and Dryer, F. L. 1981, Combustion Science and Technology, $27,31$.

12. Charles, R., Detailed Data Set: Velocity $\left(\bar{u}, \bar{v}, w_{\mathrm{rms}}\right)$ and Temperature $(\bar{T})$ Measurements in the Axisymmetric Can Combustor (ASCC) for a Parametric Variation in Inlet Conditions, University of California-Irvine Combustion Laboratory Report UCI-ARTR-87-6, 1988.

13. Charles, R. E., Emdee, J. L., Muzio, L. J., and Samuelsen, G. S., Twenty-First Symposium (International) on Combustion, The Combustion Institute, 1986, p. 1455.

14. Cloutman, L. D., "Numerical Simulation of Radiative Heat Loss in an Experimental Burner," Lawrence Livermore National Laboratory report UCRL-JC-115048, 1993; presented at the 1993 Fall Meeting of the Western States Section Meeting of the Combustion Institute.

15. Cloutman, L. D., "A Database of Selected Transport Coefficients for Combustion Studies," Lawrence Livermore National Laboratory report UCRL-ID-115050, 1993. 
Table 1. Chemical Reaction Network

\begin{tabular}{ll}
\hline \multicolumn{1}{c}{ Kinetic } & Equilibrium \\
\hline $\mathrm{CH}_{4}+2 \mathrm{O}_{2} \rightarrow \mathrm{CO}_{2}+2 \mathrm{H}_{2} \mathrm{O}$ & $\mathrm{N}_{2} \rightleftharpoons 2 \mathrm{~N}$ \\
$\mathrm{O}+\mathrm{N}_{2} \rightleftharpoons \mathrm{NO}+\mathrm{N}$ & $\mathrm{H}_{2} \rightleftharpoons 2 \mathrm{H}$ \\
$\mathrm{N}+\mathrm{O}_{2} \rightleftharpoons \mathrm{NO}+\mathrm{O}$ & $\mathrm{O}_{2} \rightleftharpoons 2 \mathrm{O}$ \\
$\mathrm{OH}+\mathrm{N} \rightleftharpoons \mathrm{NO}+\mathrm{H}$ & $\mathrm{O}_{2}+\mathrm{H}_{2} \rightleftharpoons 2 \mathrm{OH}$ \\
& $\mathrm{O}_{2}+2 \mathrm{H}_{2} \mathrm{O} \rightleftharpoons 4 \mathrm{OH}$ \\
& $\mathrm{O}_{2}+2 \mathrm{CO} \rightleftharpoons 2 \mathrm{CO}_{2}$ \\
\hline
\end{tabular}



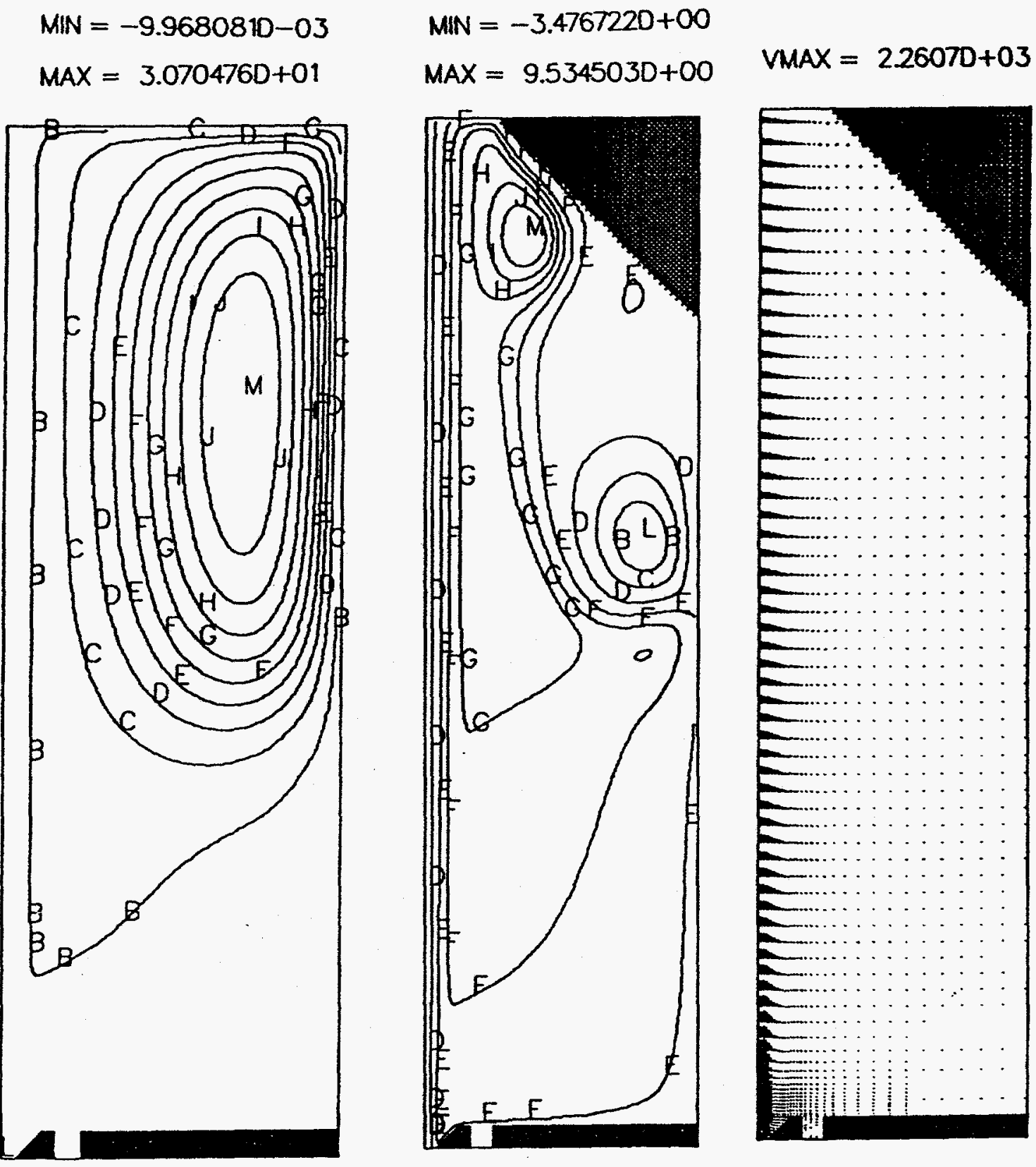

Fig. 1. Typical results of cold flow calculations with a cone-annular nozzle. The center panel is the streamline plot for the calculation made using the entire furnace. The left panel is the same problem, but with the grid truncated at the base of the sloping roof. The right panel is the velocity vector plot corresponding to the streamlines in the center panel. 


\section{VEL CYCLE $=10000 \quad$ VMAX $=1.0354 D+04$ \\ CELL CENTER INDICES $\quad 2-49,2-288$}
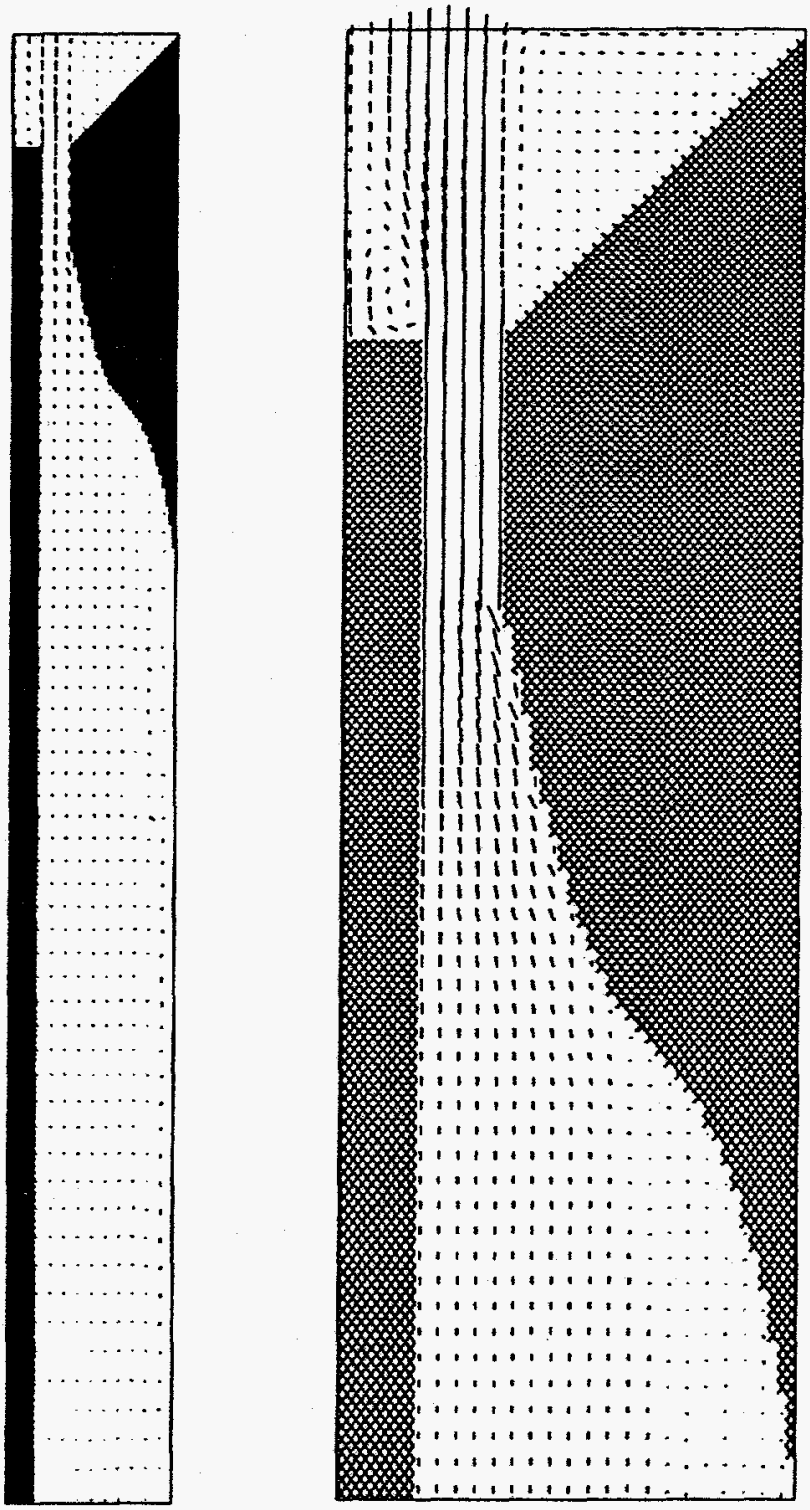

$$
\stackrel{W}{\text { MAX }}=9.0646070+03
$$

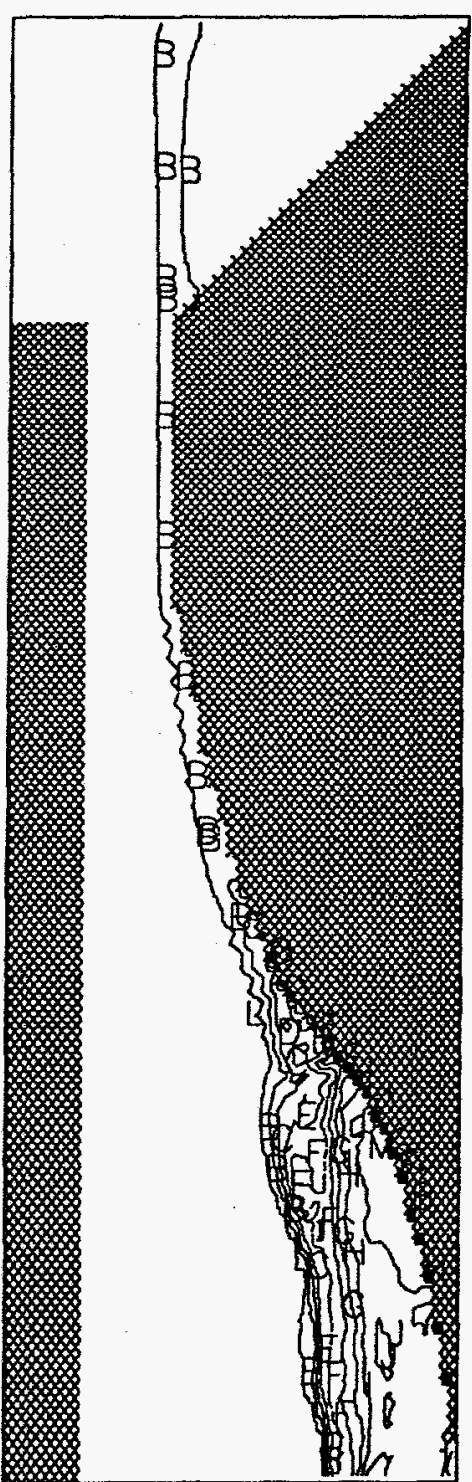

Fig. 2. Velocity plots in the swirl generator. The left plot shows the cross section of the swirl generator, with the axis of symmetry at the left and the top of the quarl at the top. The pipe supplying fuel is the obstacle lying along the axis. The center plot is a closeup of the velocity vectors in the quarl region, and the right plot shows contours of the swirl velocity. This solution is not well relaxed yet. 


$$
\operatorname{MAX}=2.2971050+03 \mathrm{MIN}=2.980179 \mathrm{D}+02 \mathrm{DQ}=1.999087 \mathrm{D}+02
$$

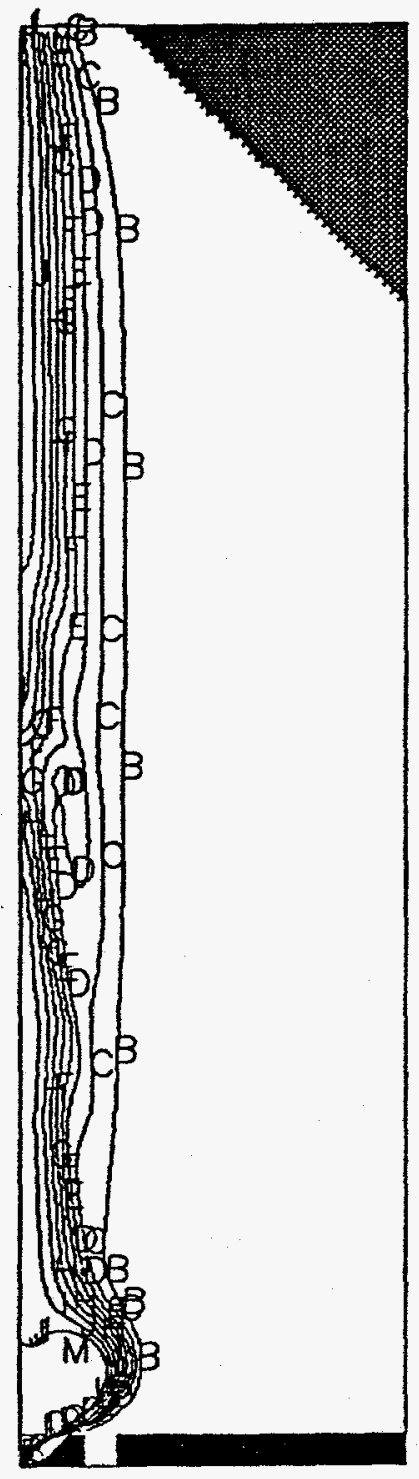

Fig. 3. Isotherms for the base case burner with a cone-annular fuel nozzle. The flame is confined to the small rounded region at the lower left-hand corner. 


$$
\operatorname{MAX}=2.297105 D+03 \mathrm{MN}=2.980179 D+02 D Q=1.9990870+02
$$

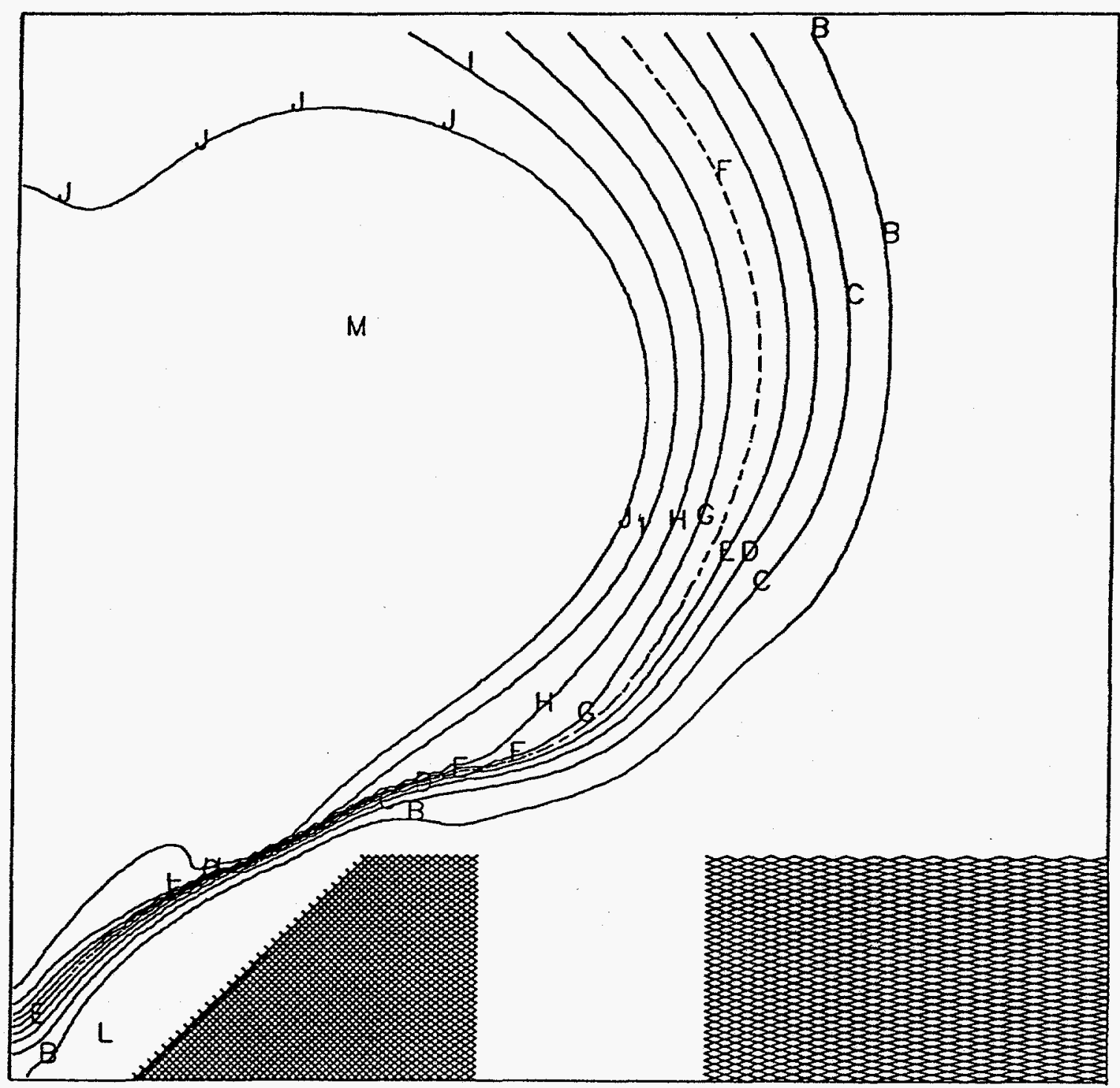

Fig. 4. Isotherms for the base case solution in the $12 \mathrm{~cm}$ square region in the lower left-hand corner of Fig. 3. 


$$
\operatorname{MAX}=8.070509 D-06 \mathrm{MIN}=-1.551354 \mathrm{D}-10 \mathrm{DQ}=8.070664 \mathrm{D}-07
$$

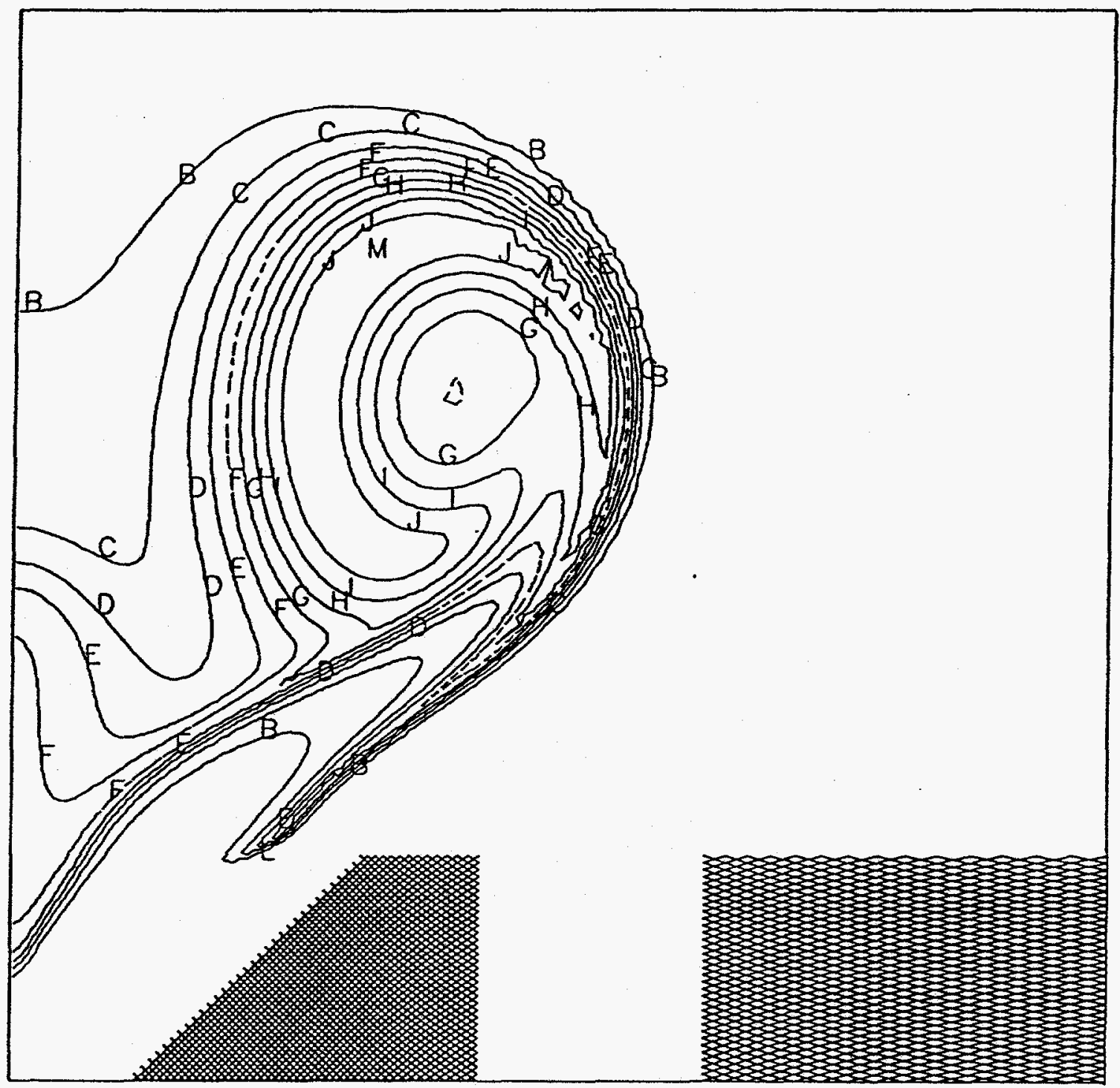

Fig. 5. The rate of chemical production of NO in the same region as shown in Fig. 4. 


$$
\operatorname{MAX}=9.979153 D-04 \quad \mathrm{MIN}=0.000000 D+00 D Q=9.979153 \mathrm{D}-05
$$

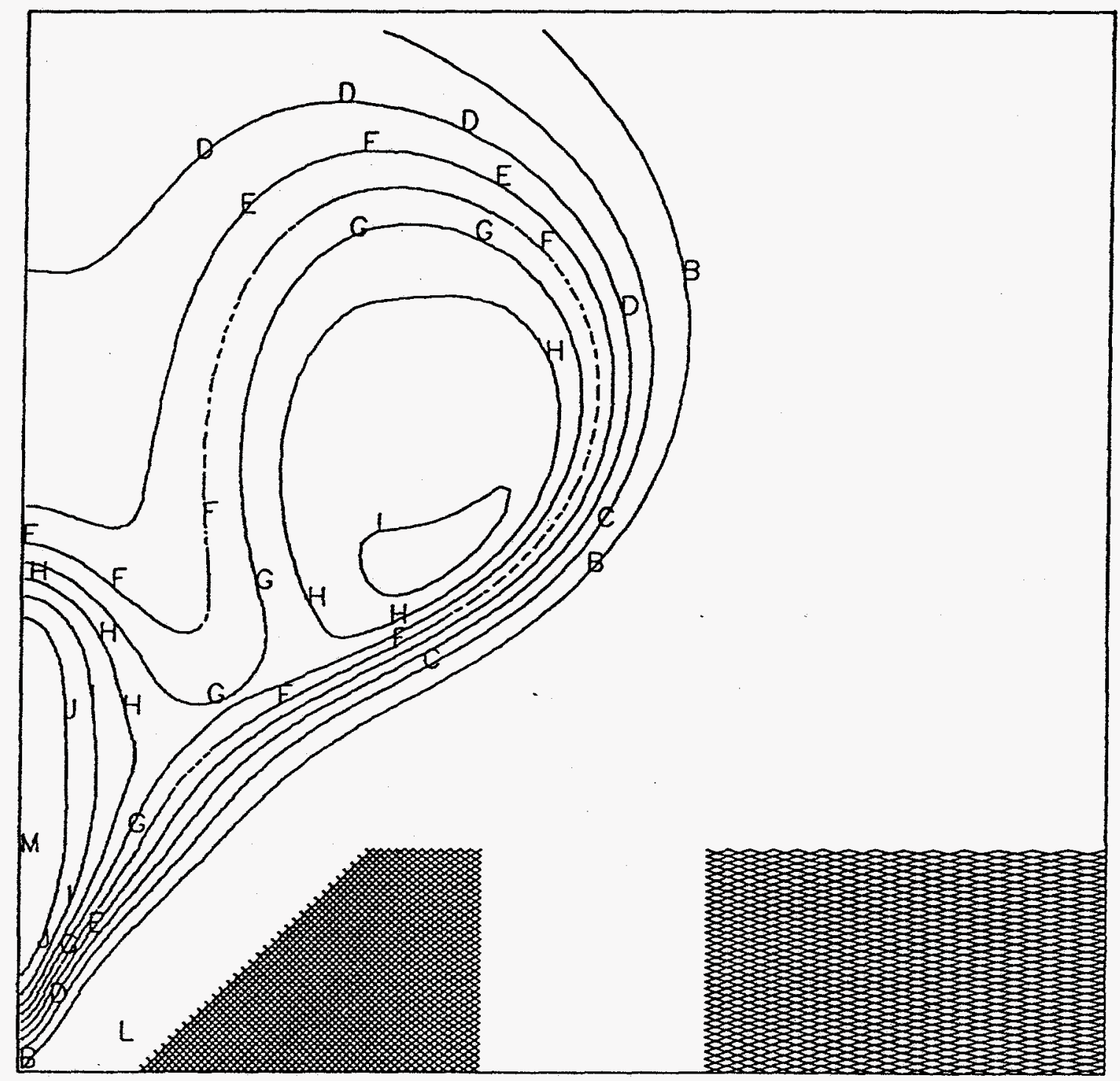

Fig. 6. NO mass fraction contours in the same region as shown in Fig. 4. 
$T=1.1182950-01$

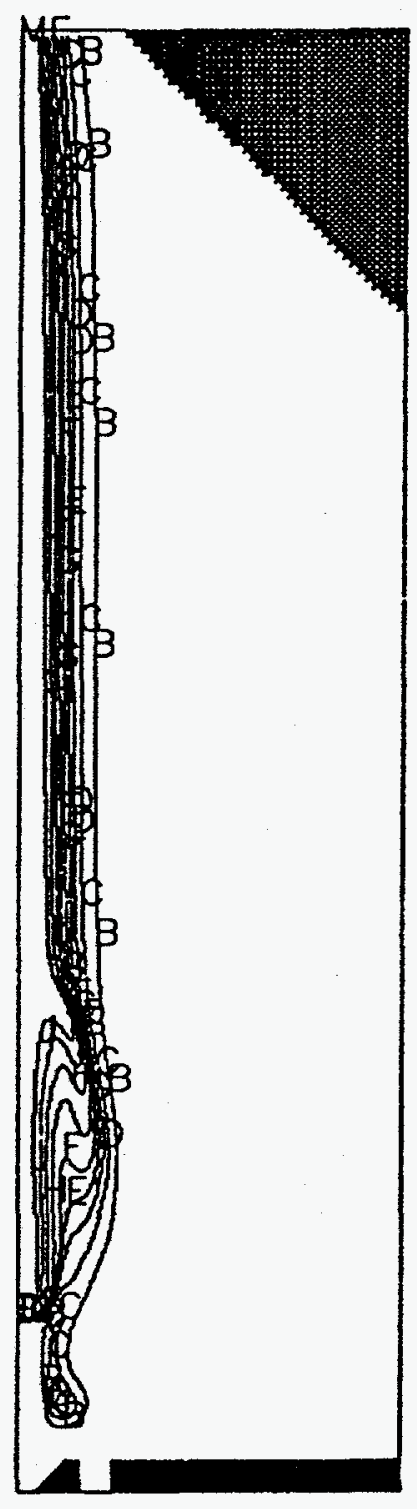

$1.7348950-01$

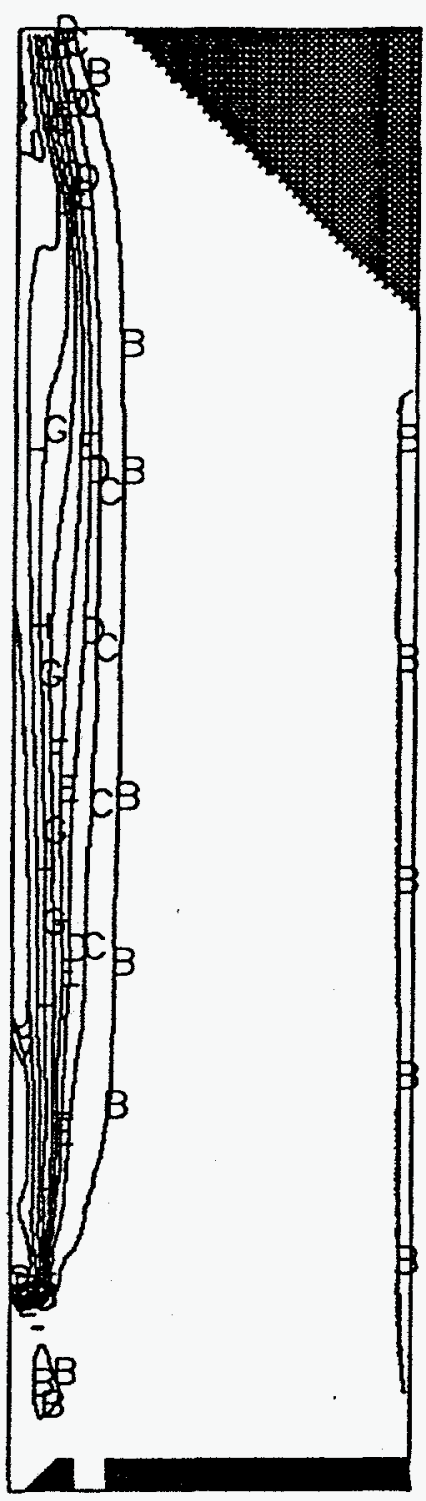

$2.1818440-01$

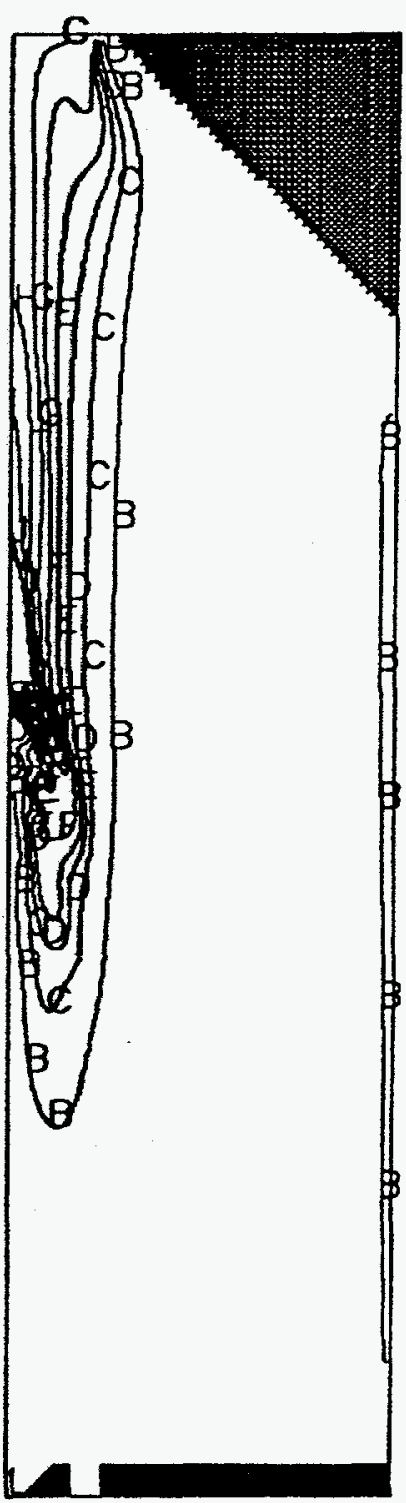

Fig. 7. Time sequence of isotherms for the cone-annular nozzle burner with no swirl. The panels are labeled at the top with the time in seconds since ignition. 


$$
M A X=2.254982 D+03 M I N=2981551 D+02 D Q=1.9568270+02
$$

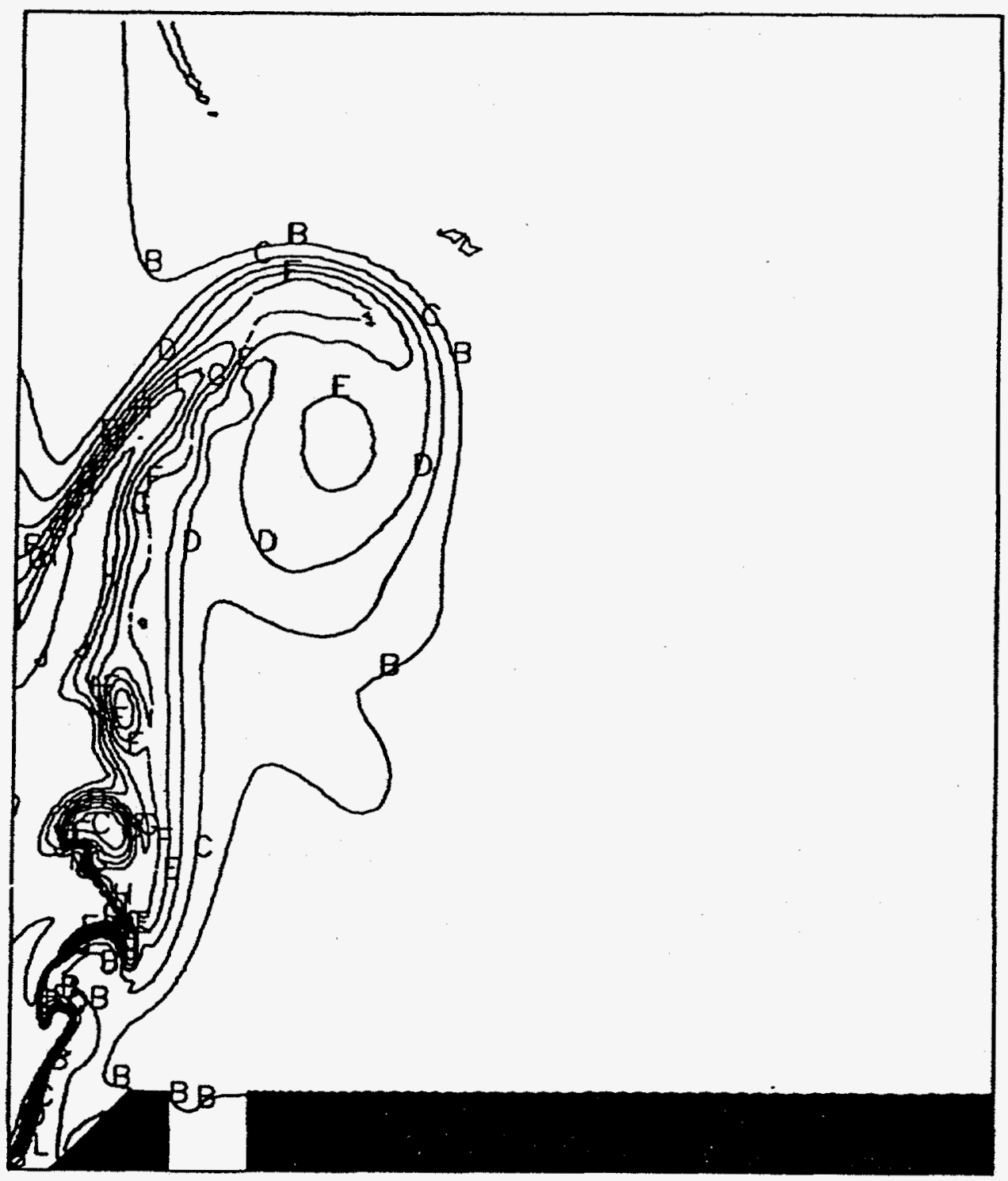

Fig. 8. Isotherms for the cone-annular nozzle with swirl and a modulated air flow rate. 


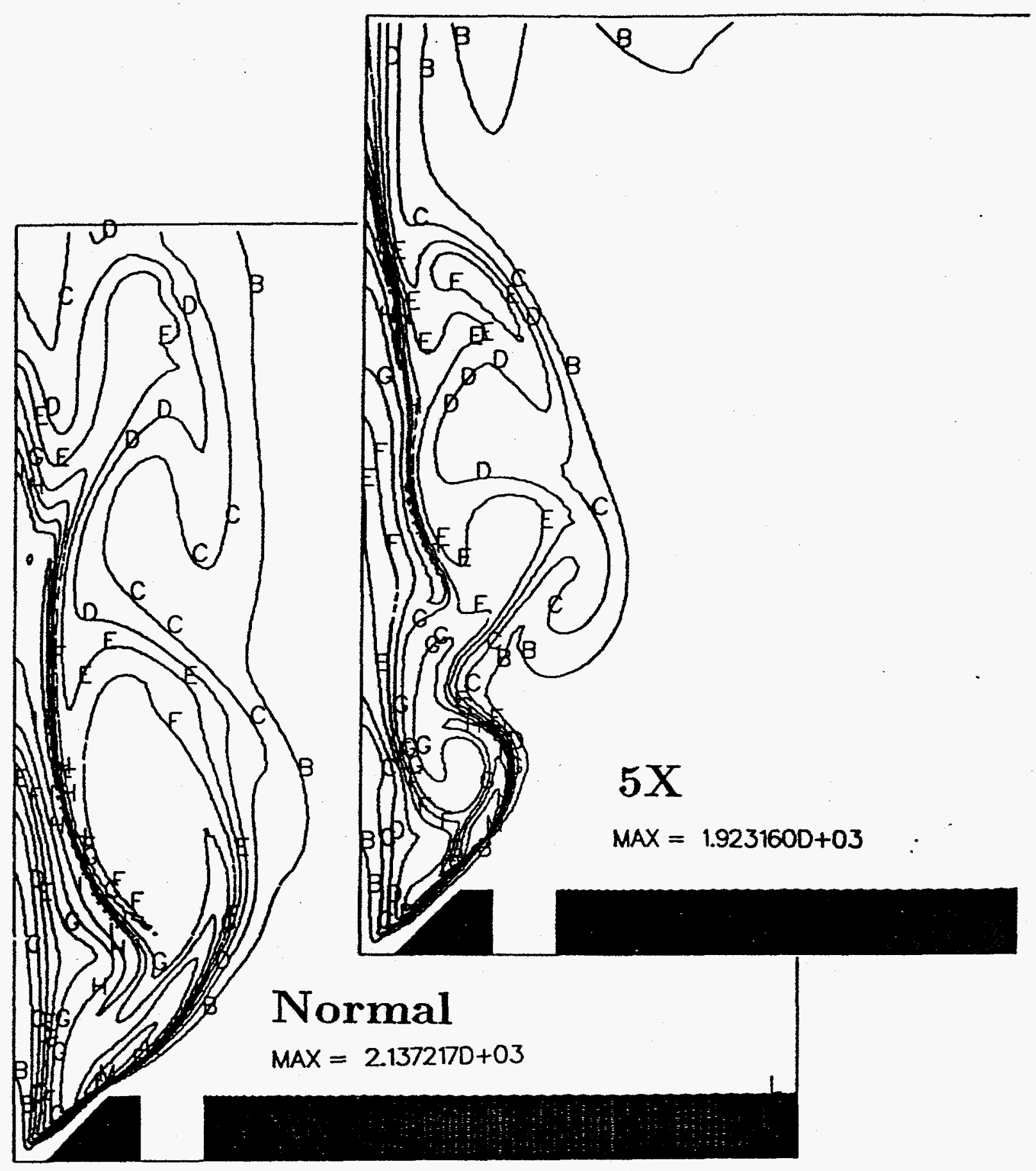

Fig. 9. Isotherms for two solutions with the axial fuel injector. The left panel is for the laboratory scale furnace. The right panel has all dimensions increased by a factor of five, and the flow speeds at the nozzle are the same. 


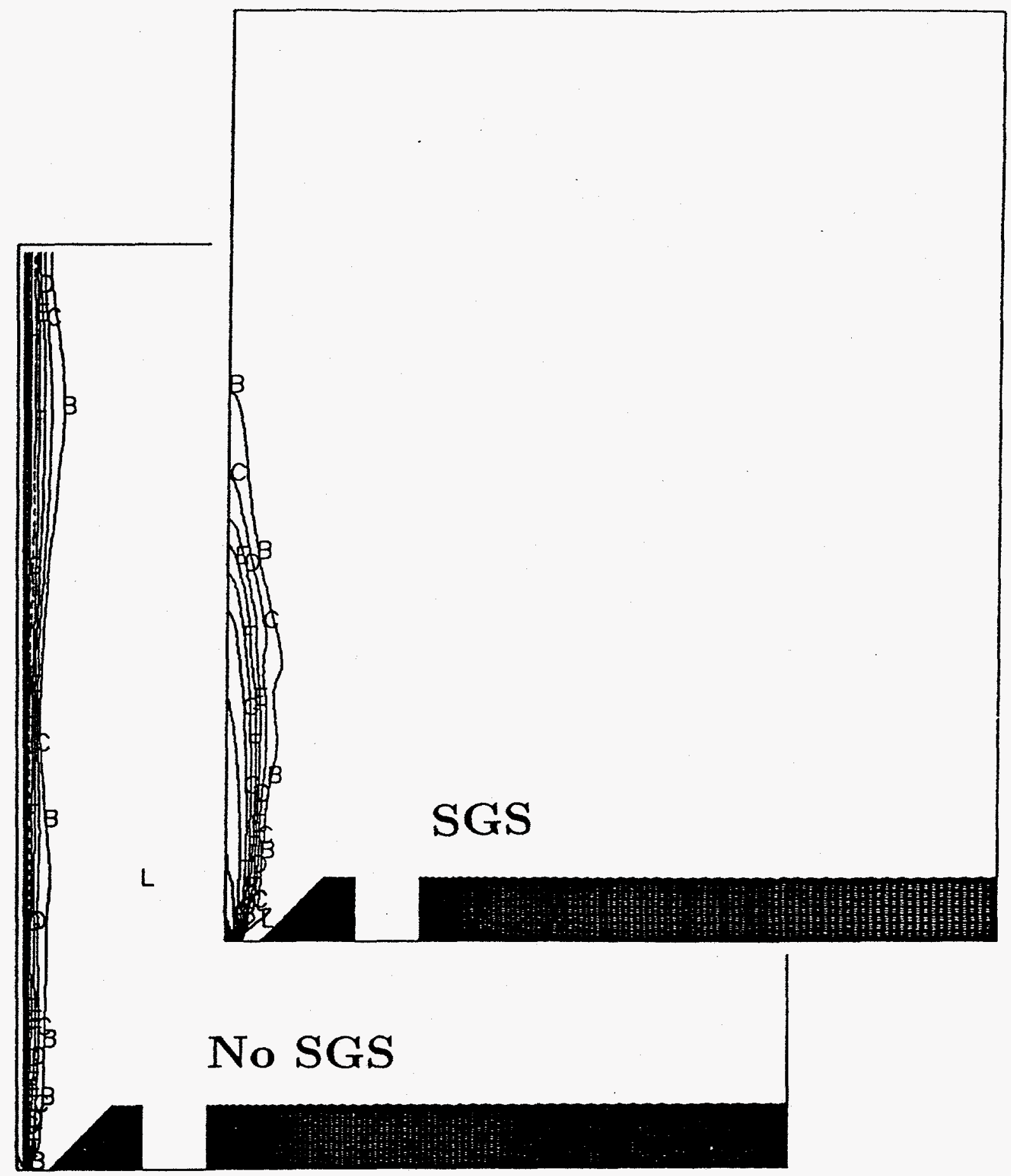

Fig. 10. Methane mass fraction contours for the axial injector, with and without the SGS turbulence model used in the calculations. 


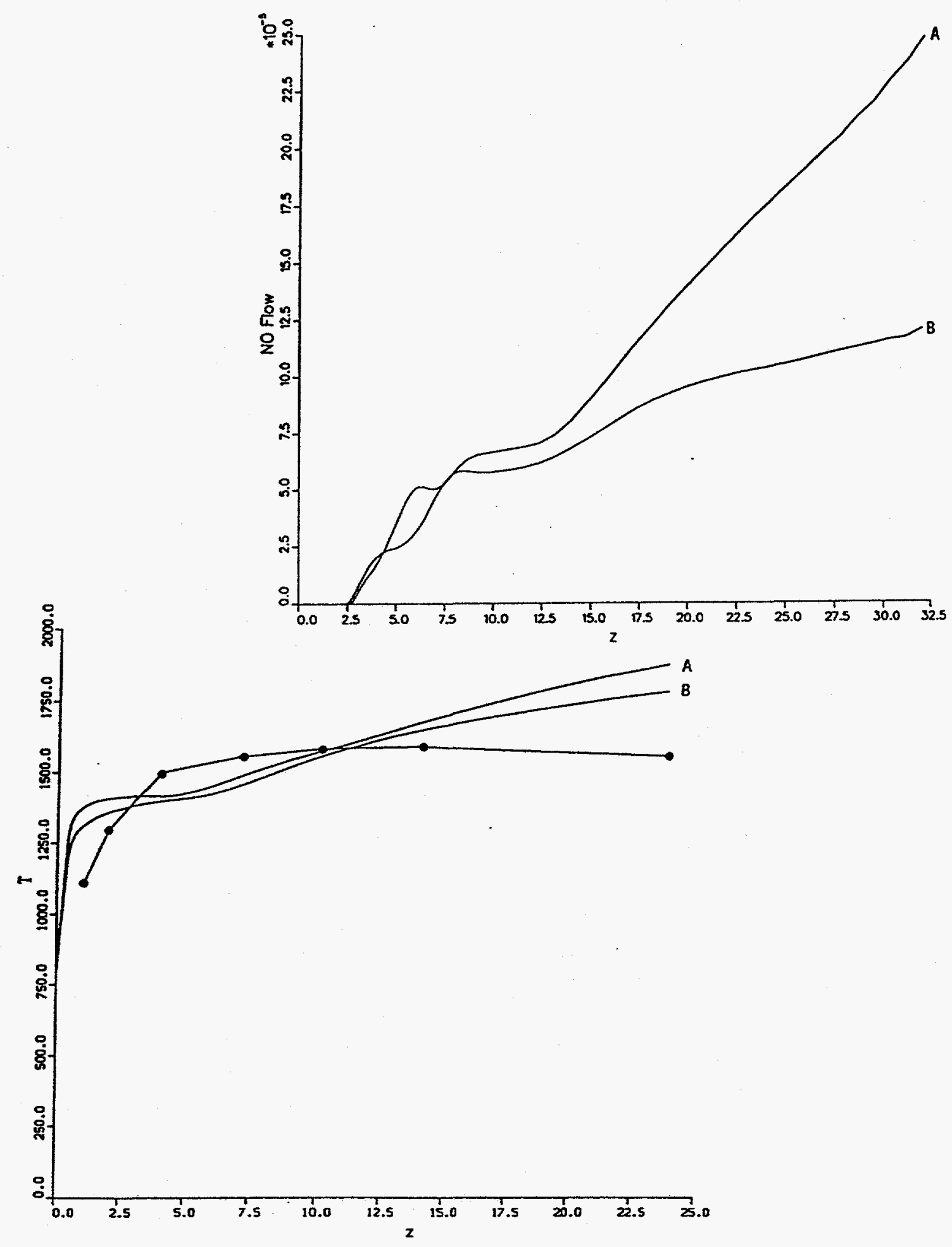

Fig. 11. Axial temperature distribution and NO mass flow rate for the Charles burner. The solid circles on the temperature plot are experimental values with an uncertainty of approximately $100 \mathrm{~K}$. Curves labelled with an $\mathrm{A}$ have no radiative losses, those with a $\mathrm{B}$ include radiation. 